\title{
Feasibility of beam crystallization in a cooler storage ring
}

\author{
Yosuke Yuri \\ Takasaki Radiation Chemistry Research Institute, Japan Atomic Energy Agency, \\ 1233 Watanuki-machi, Takasaki 370-1292, Japan
}

\author{
Hiromi Okamoto \\ Department of Quantum Matter, Graduate School of Advanced Sciences of Matter, Hiroshima University, \\ 1-3-1 Kagamiyama, Higashi-Hiroshima 739-8530, Japan
}

(Received 26 July 2005; published 7 November 2005)

\begin{abstract}
It has been known theoretically that a charged-particle beam circulating in a storage ring exhibits an "ordered" configuration at the space-charge limit. Such an ultimate state of matter is called a crystalline beam whose emittance is ideally equal to zero except for quantum noise. This paper discusses how close one can come to various ordered states by employing currently available accelerator technologies. The dynamic nature of ultracold beams and conditions required for crystallization are briefly reviewed. Molecular dynamics simulations are performed to study the feasibility of this unique phenomenon, considering practical situations in general cooling experiments. It is pointed out that several essential obstacles must be overcome to reach a three-dimensional crystalline state in a storage ring. Doppler laser cooling of ion beams is also numerically simulated to explore the possibility of beam crystallization in an existing machine.
\end{abstract}

DOI: 10.1103/PhysRevSTAB.8.114201

PACS numbers: 29.20.Dh, 52.59.Sa, 41.75.-i

\section{INTRODUCTION}

Suppose a dynamical system consisting of many identical particles of mass $m$. The average kinetic energy is given by $W=\left(\left\langle p_{x}^{2}\right\rangle+\left\langle p_{y}^{2}\right\rangle+\left\langle p_{z}^{2}\right\rangle\right) / 2 m$ where $\mathbf{p}=\left(p_{x}, p_{y}, p_{z}\right)$ is the momentum of a particle, and $\langle X\rangle$ stands for taking the average of the quantity $X$ over the whole phase space. $W$ divided by the Boltzmann constant $k_{\mathrm{B}}$ is often referred to as the temperature of the system while, more correctly, the energy of "random" motion should be averaged to evaluate the thermodynamic temperature $T$. Specifically, $W$ and $k_{\mathrm{B}} T$ are almost equal in a hot system. When the center of mass is moving at a certain speed, we define $T$ in the rest frame, subtracting the contribution from the centroid and ordered motions, so only random motion contributes. Even after such subtraction, we find that the temperature of a charged-particle beam propagating in a typical accelerator is still quite high due to the betatron and synchrotron oscillations driven by external electromagnetic forces; seen from the rest frame of the beam, individual particles randomly oscillate about the reference orbit at high speed, which means that most beams naturally have rather large energy spread in all 3 degrees of freedom and thus $W \approx$ $k_{\mathrm{B}} T$. This internal thermal energy can, however, be removed by introducing dissipative interactions into the system. As a dissipative process advances, the beam becomes denser in phase space or, in other words, the emittance is more diminished. Ideally, we can reach a "zeroemittance" state where the beam is Coulomb crystallized [1]. The space-charge repulsion of a crystalline beam just balances the external restoring force provided by electromagnetic elements such as quadrupole magnets and radiofrequency (rf) cavities.
When the beam focusing force is uniform along the reference orbit, it is possible to realize a completely "frozen" state in which $W=0$. All particles are then fixed at specific coordinates and never move in the rest frame. Schiffer and co-workers numerically studied such an ideal system, employing the molecular dynamics (MD) approach [2,3]. Particles forming a frozen beam are spatially ordered so as to achieve a perfect balance between the internal Coulomb repulsion and the beam focusing force. A question is whether an analogous crystallized state can be established in an actual accelerator where the beam is exposed to nonuniform focusing forces. This important subject was examined by Wei, Li, and Sessler who incorporated complex lattice structures into MD simulations [4]. They showed that it is still possible to form various ordered configuration, although $W$ is now nonzero (unlike uniform focusing situations). As we can readily understand, any beam must execute a breathing motion owing to the discrete nature of a modern strong focusing lattice [5]. It is thus only approximately feasible to produce a frozen beam in reality.

In this paper, we explore whether beam crystallization is practically achievable in a storage ring with the help of realistic cooling methods. For the sake of completeness, we devote Sec. II to reviewing the dynamics of coasting crystalline beams. Current understandings are described regarding the threshold densities of structural transitions, lattice conditions required for beam stability, and singleparticle orbits in a crystalline state. We then outline, in Sec. III, some essential obstacles toward beam crystallization and present related MD results that verify theoretical expectations. Emphasis is placed upon the effects of mo- 
mentum dispersion and asymmetric perturbations, both of which can seriously affect the stability of ordered structures. Advanced MD simulations of laser cooling are performed in Sec. IV to check if a phase transition to a crystalline state can be accomplished in practice. Finally, the present results are summarized in Sec. V.

\section{CRYSTALLINE BEAMS}

\section{A. Transition density}

The phase transition of a classical one-component plasma is often defined with the Coulomb coupling parameter $\Gamma_{p}$ [6]. $\Gamma_{p}$ is given by the ratio of the average Coulomb energy to the average kinetic energy; namely, $\Gamma_{\mathrm{p}}=\left(q^{2} / 4 \pi \varepsilon_{0} \bar{d}\right) / k_{\mathrm{B}} T$ where $q$ is the charge state of the particle and $2 \bar{d}$ is the average interparticle spacing. It is commonly said that a transition to a Wigner crystalline state takes place when $\Gamma_{\mathrm{p}}$ goes beyond 170 [6]. A similar definition applies to beam crystallization as long as $T$ is properly evaluated. Since the external beam confinement force varies periodically, we must carefully exclude the kinetic energy of the ordered oscillation from $k_{\mathrm{B}} T$.

Hasse and Schiffer have theoretically investigated the structural transition of infinitely long Coulomb crystals, assuming the time-independent linear potential for particle confinement [7]. According to their analysis, the spatial configuration of a crystal can be determined by the dimensionless parameter

$$
\zeta=N a_{\mathrm{wS}}
$$

where $N$ is the number of particles per unit length measured in the rest frame, and $a_{\mathrm{WS}}$ is the Wigner-Seitz radius expressed as $a_{\mathrm{WS}}=\left(3 a_{0}^{2} / 4 N\right)^{1 / 3}$ when the transverse extent of the crystal is $a_{0}$. In Fig. 1, we display typical crystalline configurations obtained from MD simulations where the lattice of the small laser-equipped storage ring (S-LSR) at Kyoto University [8] has been adopted for our numerical work. An ideal three-dimensional (3D) cooling force has been employed in these simulations to achieve complete ordering. Table I lists the structural transition densities predicted in Ref. [7]. Since this theoretical prediction is based on the harmonic potential model, it only approximately explains the actual transition of coasting crystalline structures exposed to a strong focusing force. Once a longitudinal focusing field is switched on, the ordered configuration depends also on how strongly the beam is compressed in that direction. Even if the total number of stored particles in a ring is unchanged, we can alter the crystalline structure by either strengthening or weakening the bunching force.

\section{B. Required lattice condition}

The lattice of a storage ring aiming at beam crystallization must satisfy a couple of conditions [4,9]. First of all, in order to form an ordered structure, the ring has to operate
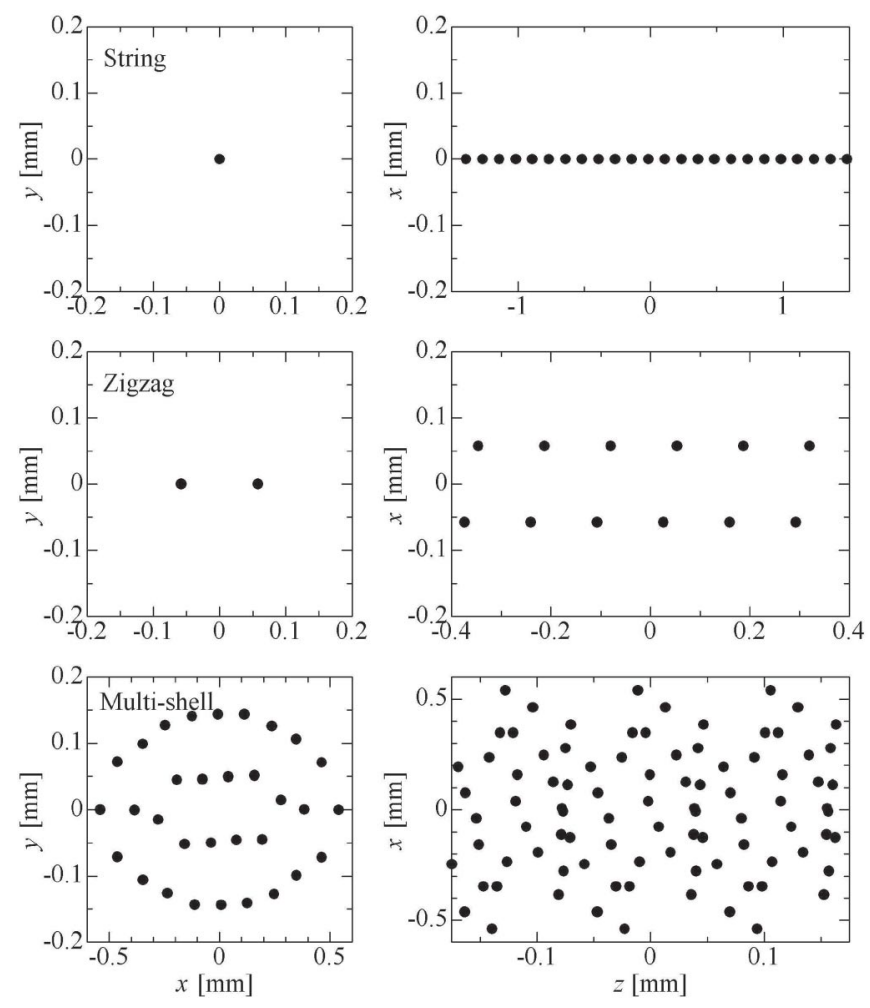

FIG. 1. Real-space configurations of typical coasting crystalline beams. The horizontal and vertical bare betatron phase advances per lattice period have been both set at 86.6 degrees in all three examples. Each dot $(\bullet)$ in the pictures corresponds to a single ${ }^{24} \mathrm{Mg}^{+}$ion circulating in S-LSR at the kinetic energy of $35 \mathrm{keV}$. The symbols $(x, y, z)$ represent, respectively, the horizontal, vertical, and longitudinal spatial coordinates in the beam rest frame. At very low line density, a one-dimensional (1D) string crystalline structure is formed (upper panel). By increasing $N$, we can transform a string into a two-dimensional (2D) zigzag crystal (middle panel) and, then, eventually attain a 3D shell crystal (lower panel). These ordered structures are stable without dissipation.

below the transition energy $\gamma_{\mathrm{T}}$. It is thus impossible to crystallize a beam in a weak focusing machine. Second, the following is required to maintain a crystalline beam:

$$
2 \sqrt{2} \max \left(\nu_{x}, \nu_{y}\right)<N_{\mathrm{sp}}
$$

where $\nu_{x}$ and $\nu_{y}$ are the horizontal and vertical betatron

TABLE I. Transition densities of coasting Coulomb crystals confined in the time-independent potential [7].

\begin{tabular}{lc}
\hline \hline \multicolumn{1}{c}{ Scaled density } & Crystal structure \\
\hline $0<\zeta<0.709$ & String (1D) \\
$0.709<\zeta<0.964$ & Zigzag (2D) \\
$0.964<\zeta<3.10$ & Single shell (3D) \\
$3.10<\zeta<5.7$ & Single shell + string (3D) \\
$5.7<\zeta<9.5$ & Double shells (3D) \\
\hline \hline
\end{tabular}


tunes, and $N_{\mathrm{sp}}$ is the lattice superperiodicity of the ring. This is the condition to keep all phonon modes away from linear resonance with the periodic driving force $[4,10]$. According to a recent simulation study [11], the maintenance condition (2) is necessary but may not be sufficient from a practical point of view. We will discuss this matter in detail later.

The two requirements above can easily be met in a compact ion trap system. Coulomb crystalline states similar to the numerical examples in Fig. 1 have already been realized in many ion traps around the world by using the laser-cooling technique [12-14]. This advanced technique is currently the only means for us to generate Coulomb crystals because of its high damping rate and very low limiting temperature. Considering a physical analogy between a Paul trap and a linear beam transport [15], it should be possible, at least in theory, to crystallize even a highenergy ion beam if its orbit is linear. Laser cooling is, however, applied only to a circulating beam in a storage ring, so that each ion scatters many photons in one or more straight sections every turn $[16,17]$. Because of this technical requirement for extending the effective cooling region, we are forced to introduce bending magnets that make the beam orbit closed. As discussed in Sec. III, the dipole fields peculiar to a circular machine play a crucial role in crystalline states.

\section{Single-particle orbit in a crystalline state}

In a coasting crystalline beam as displayed in Fig. 1, the trajectories of all particles are proportional to each other [18]. The transverse motion of any single particle in the beam can be expressed, with universal orbit functions $D_{x}$ and $D_{y}$, as

$$
x=C_{x} D_{x}, \quad y=C_{y} D_{y},
$$

where $C_{x(y)}$ is a scaling constant that depends on which particle we see. It has been proven that $C_{x}$ is equivalent to the momentum deviation $\delta p / p$ of each particle [18]. $D_{x}$ and $D_{y}$ satisfy coupled differential equations similar to the Kapchinsky-Vladimirsky envelope equations for a zeroemittance beam [18]. The 3D crystalline beam thus breathes as a whole responding to external focusing forces. The periodic particle oscillations driven by an alternating gradient lattice make the average kinetic energy of the crystal finite. In the lower case of Fig. 1, the energy of the breathing motion is a few Kelvin that is much higher than the Doppler cooling limit. This value becomes greater and greater as the number of shells increases at higher line density.

The horizontal (vertical) emittance is usually defined as the area occupied by the beam in $x-x^{\prime}\left(y-y^{\prime}\right)$ phase space, where $x^{\prime} \equiv d x / d s\left(y^{\prime} \equiv d y / d s\right)$. Equations (3) indicate that all particles have the same $x^{\prime} / x$ and $y^{\prime} / y$ at any location of the ring, which implies that the phase-space distribution is always a straight line as shown in Fig. 2. The
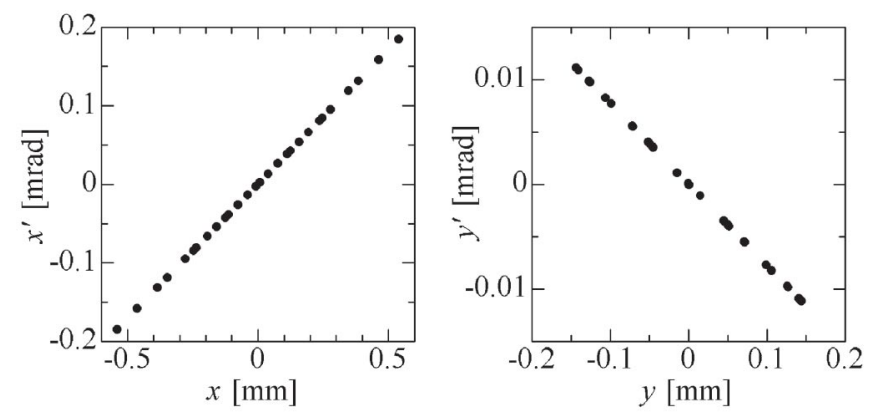

FIG. 2. Phase-space configuration of the double-shell crystalline beam in Fig. 1. The linear distribution is maintained all around the storage ring, although the tilt angle changes periodically according to the function $\left(d D_{x(y)} / d s\right) / D_{x(y)}$. If the focusing force is uniform along the orbit, all particles are aligned on the $x^{\prime}=0$ and $y^{\prime}=0$ axes (thus, $W=0$ ) and never move.

transverse emittance of a crystalline beam is, therefore, zero regardless of its configuration in real space. This is true no matter whether the crystal is continuous or bunched. We can thus state that crystalline beams have achieved the lowest possible emittance that a beam may have.

\section{OBSTACLES TO BEAM CRYSTALLIZATION}

\section{A. Intrabeam scattering}

Even if the two necessary conditions in Sec. II B are satisfied, crystallizing a beam is not always possible in practice. There are additional undesirable factors that can seriously disturb a cooling process toward crystallization. First of all, intrabeam scattering starts to dominate the beam as the emittance is more diminished. If the cooling efficiency is too low, the beam will settle into a sort of equilibrium at a rather high temperature determined by the balance between the internal heating and external damping forces. We expect that the heating rate comes to a peak in the liquid phase where the average Coulomb potential is on the same order as the thermal energy $k_{\mathrm{B}} T$. Once the beam goes beyond the peak, the heating due to random Coulomb collisions becomes less dangerous and eventually disappears in a perfect crystalline state [9]. The cooling force must, therefore, be strong enough to overcome the heatingrate mountain. Figure 3 shows two MD results in which identical simulation parameters have been assumed except for the cooling strength. In the upper pictures, the momentum of each particle is reduced by $1 \%$ every lattice period while the friction has been doubled in the lower pictures. Only a $1 \%$ increase of the cooling strength has made the final beam state essentially different. Note that this effect does not take place in a spatially uniform lattice.

\section{B. Coherent resonance}

Another critical effect that may limit the achievable beam emittance is the so-called coherent resonance. 


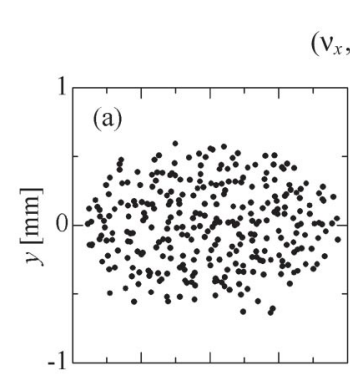

$\left(v_{x}, v_{y}\right)=(1.444,1.444)$
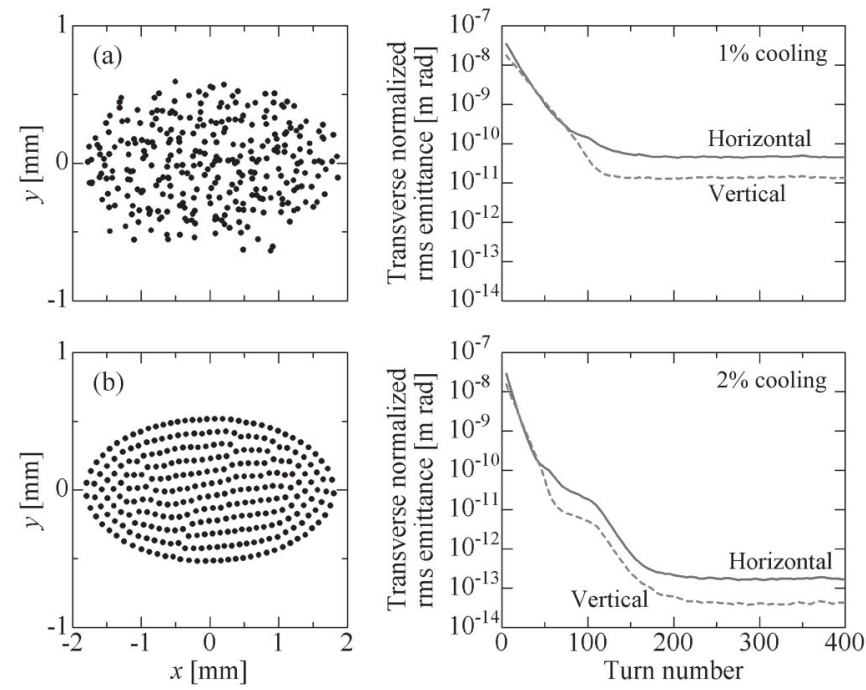

FIG. 3. MD results of two ion beams cooled with a linear frictional force. The 3D cooling force has been slightly strengthened in the lower pictures, compared to the upper case. The lattice parameters are the same as those assumed in Fig. 1. The beam is continuous and the line density is $2.5 \times 10^{6} \mathrm{~m}^{-1}$.

Equation (2) can actually be interpreted as a sufficient condition to avoid the occurrence of the linear coherent resonance at the space-charge limit. This suggests that we should be careful in applying Eq. (2) because ordinary beams are far from the space-charge limit before cooling; there is no guarantee as to whether the beam can reach a crystalline state starting from a usual high-temperature regime. A problem is that the effective operating point of the ring moves toward the origin on the tune diagram as the beam density becomes higher; the actual oscillation frequency of each particle gradually decreases due to the increasing Coulomb repulsion during a cooling process (see Fig. 12). Consequently, the beam inevitably crosses many stop bands lying between the initial and final operating points. Recent $2 \mathrm{D}$ particle-in-cell simulations have revealed that a beam of high line density cannot go across a linear-resonance stop band even under the influence of a strong cooling force [11]. Once the lowest-order resonance is excited, the coherent tune of the beam is locked around a certain value and no further cooling can be done. A similar phenomenon has been observed experimentally in a European storage ring [19]. In order to suppress the possibility of linear-resonance crossing over the whole temperature region, the betatron phase advance per single focusing period must be taken below $\pi / 2$ (rather than $\pi / \sqrt{2}$ ) [20$22]$. Whenever the phase advance per lattice period exceeds 90 degrees, the beam will eventually encounter a severe resonance stop band before an ordered state is reached. Figure 4 is a MD result that supports this expectation. The betatron tunes have been increased from $\nu_{x}=$ $\nu_{y}=1.444$ assumed in Figs. 1 and 3 to $\nu_{x}=\nu_{y}=1.80$. These tunes still satisfy the maintenance condition (2), but

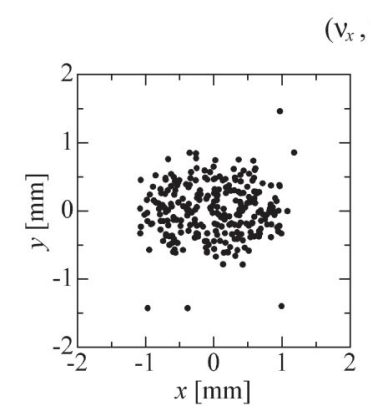

$\left(v_{x}, v_{y}\right)=(1.80,1.80)$

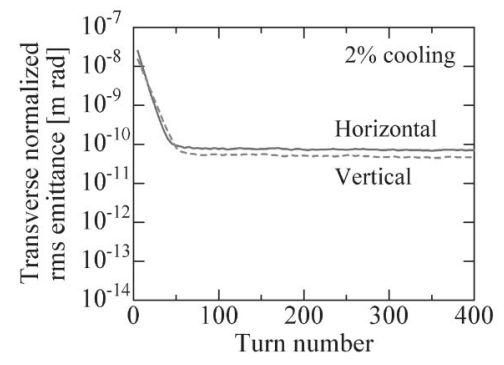

FIG. 4. MD result of an ion beam cooled with a linear frictional force. The simulation parameters are the same as those in Fig. 3 except that the transverse bare tunes have been raised to $\left(\nu_{x}, \nu_{y}\right)=(1.80,1.80)$. The strength of the friction is identical to that employed in case (b) of Fig. 3. The multishell crystal is no longer formed with these tunes whereas the maintenance condition (2) has been satisfied.

not the linear-resonance-free condition

$$
4 \max \left(\nu_{x}, \nu_{y}\right)<N_{\mathrm{sp}} .
$$

Although the other parameters including the cooling strength are unchanged, no ordering has been achieved. Figure 5 summarizes MD results obtained with various tunes and cooling rates. The line density of the beam has been fixed at $2.5 \times 10^{6} \mathrm{~m}^{-1}$ in all these simulations. This

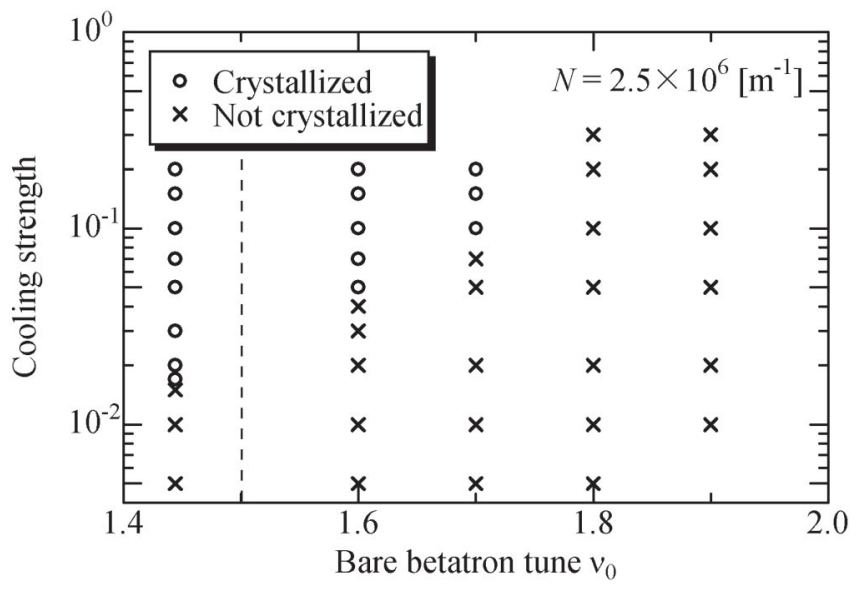

FIG. 5. Required cooling strength for beam crystallization vs transverse tune. The simulation parameters are the same as those assumed in Fig. 3 except for the betatron tunes and cooling strength. For the sake of simplicity, the horizontal and vertical tunes have been set equal in all MD simulations, i.e., $\nu_{x}=\nu_{y}(\equiv$ $\nu_{0}$ ). The beam is cooled with a $3 \mathrm{D}$ linear frictional force that has identical cooling rates in the three directions. In the region $\nu_{0}>$ 1.8 , a crystalline state cannot be reached at this line density even if the friction is considerably enhanced. Even when $\nu_{0}<1.5$, it is difficult to achieve crystallization with a low cooling rate because of the heating from intrabeam scattering (see Fig. 3). Note that the lattice of S-LSR has sixfold symmetry $\left(N_{\mathrm{sp}}=6\right)$. The vertical broken line in the picture indicates the threshold tune determined by Eq. (4). 
diagram indicates that beam crystallization becomes more and more difficult as the transverse tune increases. We thus conclude that 3D crystalline states cannot generally be established with a realistic cooling force unless the ring satisfies the condition (4).

Since this condition is relevant only to a regular beam with finite transverse dimensions, 1D and 2D crystalline beams may be free from it. Past MD simulations have actually indicated that both string and zigzag states can be stable above the present threshold. Similarly to the collisional heating, this instability does not occur provided that the external force is uniform.

\section{Dispersive effect}

So far, nobody has succeeded in crystallizing an ion beam in a storage ring [23]. There are several primary reasons for that. First, laser cooling is inefficient in damping the transverse betatron oscillations of stored ions. Second, a storage ring is much more complex than compact ion traps: magnetic field imperfections and other noise sources are inevitable which may give rise to beam heating. Third, the effects of momentum dispersion caused by dipole fields are present in a circular system. This third factor has made beam crystallization extremely difficult to achieve in practice.

The problem is, in one word, that dipole fields couple the transverse and longitudinal motions of particles. The path length of each particle depends on the horizontal coordinate $x$ in a crystalline state whenever the beam orbit is closed; a radially outer particle at larger $x$ has to travel a longer distance than inner particles every turn. In the meanwhile, all particles must have an identical revolution frequency in order for the ordered configuration to be maintained. The average longitudinal velocities are, therefore, slightly different depending on their horizontal positions. A regular cooling force, designed to simply equalize the longitudinal momenta of stored particles, is not suitable for the dispersive character of a crystalline beam. In order to stabilize an ordered structure, we need to develop a "tapered" force represented as $[9,24]$

$$
\Delta\left(\frac{\delta p}{p}\right)=-f_{z}\left(\frac{\delta p}{p}-\gamma C_{x z} \frac{x}{\rho_{0}}\right),
$$

where the left-hand side denotes the change in $\delta p / p$ before and after the cooling section, $\gamma$ is the Lorentz factor, $\rho_{0}$ is the radius of curvature in the bending regions, $f_{z}$ corresponds to the strength of the damping force, and $C_{x z}$ is called the tapering factor determined by the lattice design. Tapered cooling is a key to form a circulating crystalline beam with a finite horizontal extent [25]. Such a special force becomes unnecessary only in a dispersion-free system. A possible scheme to eliminate dispersive effects was first proposed by Pollock in Ref. [26]. Later, Ikegami et al. studied the same scheme in more detail with a general Hamiltonian formalism [27]. The idea has been incorpo- rated in S-LSR where an electrostatic deflector is inserted in every bending region to control the linear momentum dispersion [8].

Once we switch on an rf cavity in a storage ring, a crystalline beam comes to show even more unique behavior that can never be reproduced in an ion trap [28]. Since the rf field accelerates or decelerates particles, the transverse motion of a crystalline beam is influenced by the energy modulation via the dispersive coupling from bending magnets. Then, even a string crystal can no longer stay on the reference orbit but horizontally oscillates about it [29]. Figure 6 exhibits a typical bunched Coulomb chain in a storage ring. 2D and 3D bunched crystalline beams also execute analogous "head-tail" oscillations driven by momentum dispersion. The stability property of crystalline beams in a storage ring is thus much more complicated than that of "regular" Coulomb crystals in a linear ion trap. The ring-shaped Paul trap system "PALLAS" constructed by German researchers [30] may enable us to make a systematic study of these important subjects on beam crystallization. Since dispersion exists in such a circular system, a usual "untapered" laser will heat up circulating Coulomb crystals unless the shear force is sufficiently weak; we expect that the maximum shear per single focusing period should be less than the average interparticle spacing [31]. In fact, recent PALLAS experiments have suggested an instability mechanism for which the bending shear is probably responsible [32].

\section{Asymmetric perturbation}

Notice that the condition (4) can be met only approximately. Strictly speaking, the superperiodicity of any storage ring is unity because of error fields and various insertion elements. An important question arises then; namely, what degree of symmetry breakdown is acceptable in attaining a crystalline beam? Sensitivity studies have pointed out that magnetic imperfection at less than $0.1 \%$ level can be tolerated [33]. Resonances induced by error fields are, therefore, not so severe provided that dipole and quadrupole magnets are well built and well aligned. On the
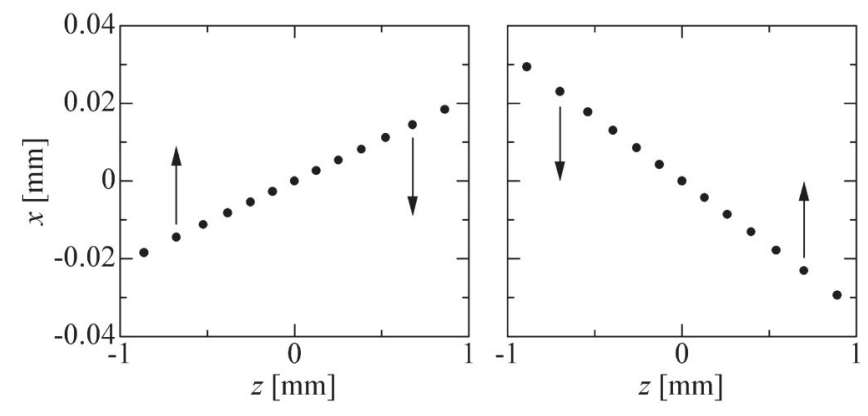

FIG. 6. Example of a bunched string crystalline beam in the storage ring S-LSR. The top views at two different locations of the ring have been depicted. The synchrotron tune is 0.07 in the absence of space charge. 
other hand, attention must be paid to the facts that we usually cool a beam in one or two straight sections and also that rf cavities are put only in limited lattice periods. As a result, additional symmetry breakdown is caused in the external forces felt by the beam. These sources of asymmetry, which are generally negligible at high temperature, start to play an important role in the ultracold regime. We have confirmed, through a number of MD simulations, that it is almost hopeless to ensure the stability of a multishell crystalline beam unless the cooling force is properly tapered and $N_{\text {sp }}$ cavities are symmetrically placed around the ring. In contrast, $1 \mathrm{D}$ and 2D crystalline beams have been found relatively insensitive to the asymmetric perturbations.

The optimum tapering factor can be evaluated from the formula $C_{x z}=\rho_{0} / \gamma D_{x}$ [18]. Since $D_{x}$ is a function of $s$ in a strong focusing machine, $C_{x z}$ varies along the design orbit. For instance, it ranges from 0.31 to 0.36 in a straight section of S-LSR when $\left(\nu_{x}, \nu_{y}\right)=(1.444,1.444)$. Needless to say, the $s$ dependence of $C_{x z}$ makes it even more difficult to actually develop an adequate tapered force. The MD results in Fig. 7 illustrate the importance of optimized tapered cooling. A "thin" tapered force has been employed here whereas a cooling region always extends over some distance in reality. Ideally, we do not have to do tapered cooling in every lattice period because the right hand side of Eq. (5) automatically vanishes after crystallization, which means that this special dissipative force has no effect in an ordered state. In case (a), we have applied an ideal tapered force once in every turn [34]. The 3D crystalline beam attained is quite stable and the ordered configuration lasts for many turns even after the cooling force is removed. The normalized root-mean-squared (rms) emittance is on the order of $10^{-13} \mathrm{~m} \mathrm{rad}$ in both transverse directions. MD results in the middle and lower pictures demonstrate what happens when the tapering factor is deviated from the optimum value. Though the resultant beams in cases (b) and (c) look still ordered (left panels), they are unstable and blow in a few hundred turns without the dissipation (right panels). These simulations convince us that it is impossible to make a multishell crystal with regular laser cooling as long as the beam is exposed to strong momentum dispersion.

As mentioned in the last subsection, the energy modulation caused by an $\mathrm{rf}$ cavity complicates the transverse motion of an ordered beam. All kinds of bunched crystals are forced to perform periodic head-tail oscillations as identified in Fig. 6. Nevertheless, it is still possible to produce a large 3D crystalline beam with tapered cooling if cavities are sitting in all lattice periods symmetrically. We give, in Fig. 8(a), an example of a bunched multishell crystal whose axis oscillates periodically on the horizontal plane. Six cavities have been switched on so that the bare synchrotron tune $\nu_{z}$ is 0.15 . The oscillation pattern of the ellipsoid axis is identical to that of a bunched

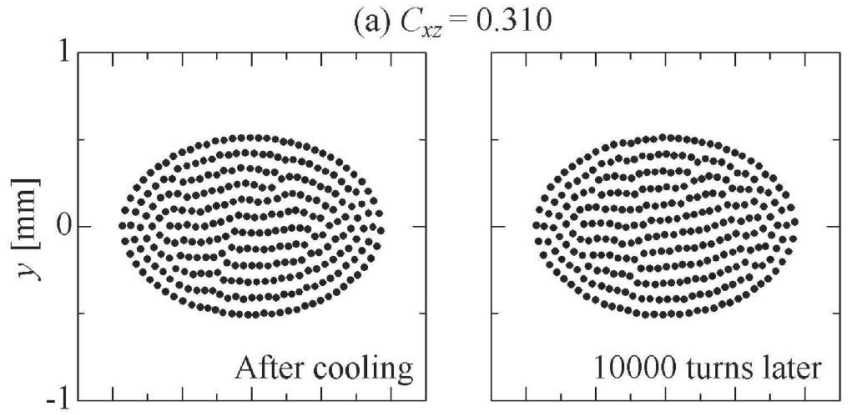

(b) $C_{x z}=0.326$

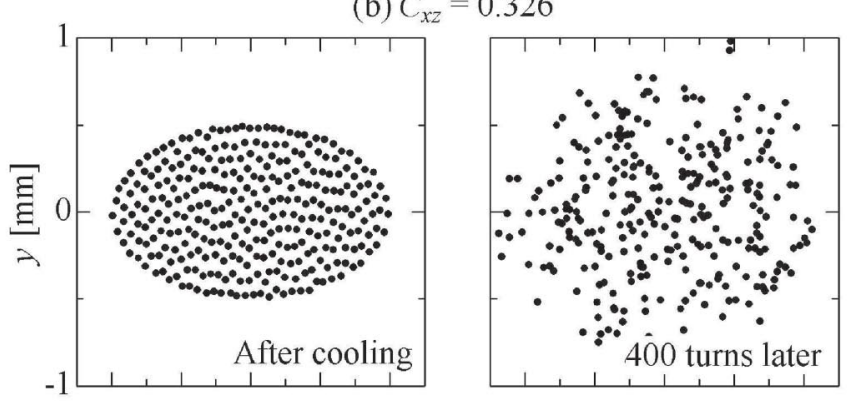

(c) $C_{x z}=0.295$
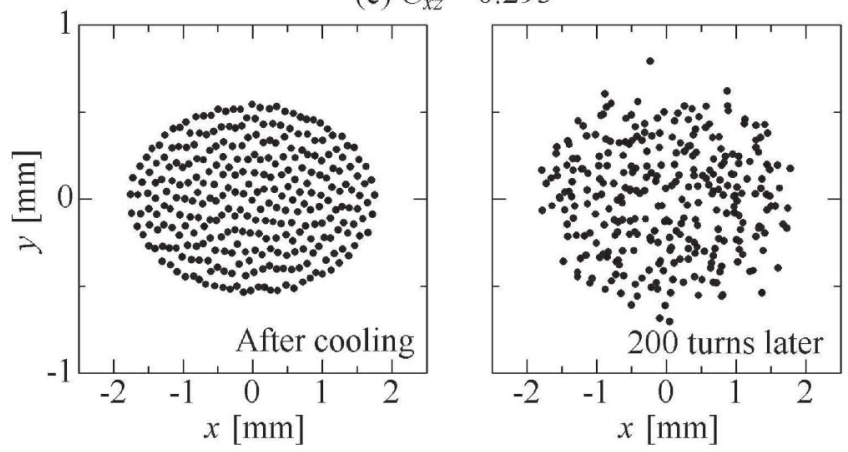

FIG. 7. Cross-sectional views of ultralow-emittance ${ }^{24} \mathrm{Mg}^{+}$ beams circulating in S-LSR at the kinetic energy of $35 \mathrm{keV}$. The transverse bare tunes have been set at $\left(\nu_{x}, \nu_{y}\right)=$ $(1.444,1.444)$, such that the condition (4) is fulfilled. We have applied a thin tapered force and weak transverse dissipation in a certain lattice period to cool a coasting beam whose line density is $2.5 \times 10^{6} \mathrm{~m}^{-1}$. The friction coefficient $f_{z}$ is 1.0 (full cooling). The tapering factor has been adjusted to the following value: (a) 0.310 (optimum), (b) 0.326 ( $+5 \%$ error), and (c) $0.295(-5 \%$ error). In order to improve the beam stability, we have gradually weakened the cooling forces to zero in 2000 turns. This procedure generally makes the survival time of a crystalline structure considerably longer. The left panels show the beam configuration at the moment when the cooling forces are damped away.

Coulomb chain formed at much lower line density; it is thus predictable with the theory in Ref. [29]. Turning off five of the six cavities, we find that the maintenance of the ordered configuration is no longer possible [Fig. 8(b)]. Since the sixfold symmetry of the longitudinal force has been broken, the period of the head-tail oscillation agrees not with the magnetic lattice period but with the ring circumference. 

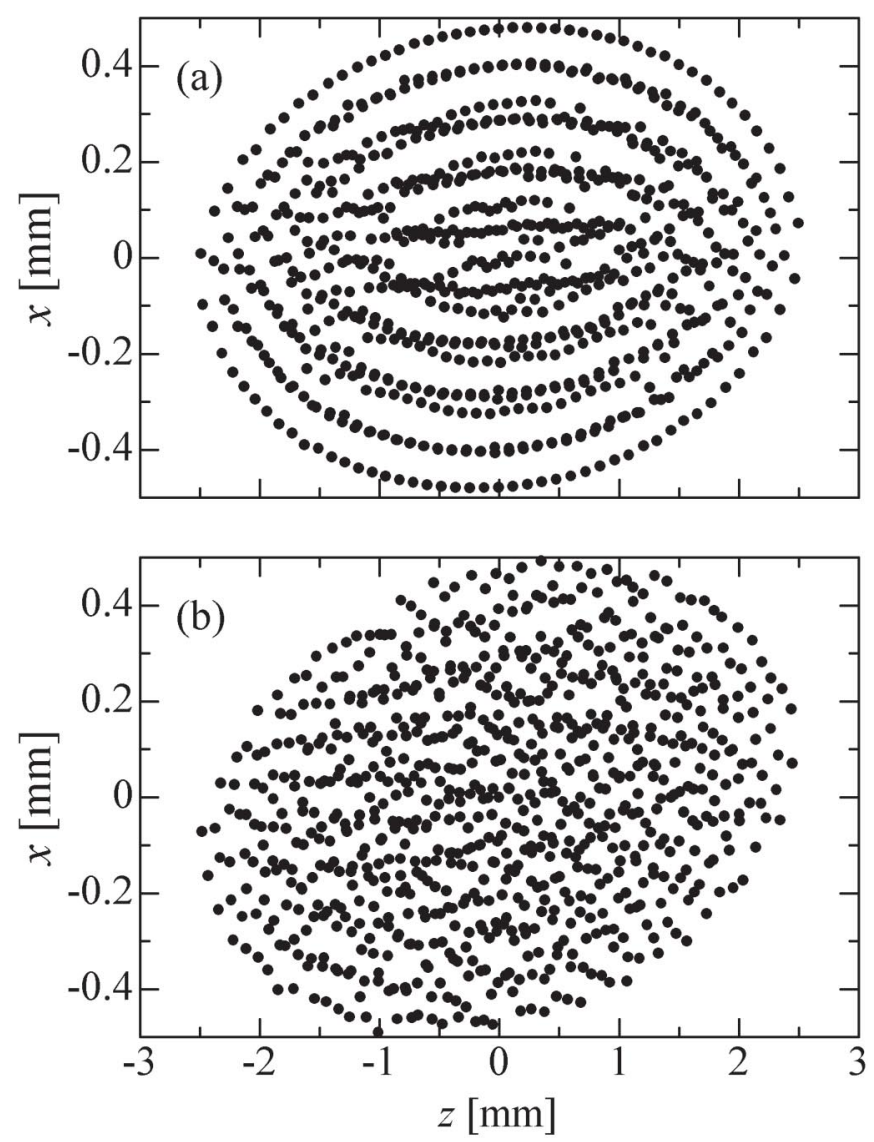

FIG. 8. Top views of ultralow-emittance beams in S-LSR. The fundamental lattice parameters are the same as those assumed in the previous figures except that we have now switched on $\mathrm{rf}$ fields to bunch the beam. The number of ${ }^{24} \mathrm{Mg}^{+}$ions in a bunch is 800 in these simulations. Case (a) corresponds to the situation where we have turned on six rf cavities symmetrically set around the ring. The amplitude of the rf voltage has been chosen to be $48.8 \mathrm{~V}$. In case (b), five of them have been switched off. In order to keep the synchrotron tune unchanged, the rf amplitude has been increased to $272 \mathrm{~V}$.

\section{3D LASER COOLING}

\section{A. The resonant coupling method}

We are now in a position to discuss the experimental feasibility of beam crystallization. Since it is not difficult to construct a machine that simultaneously fulfills the conditions (4) and $\gamma<\gamma_{\mathrm{T}}$, the most essential issue is how to develop a proper $3 \mathrm{D}$ cooling force. For this purpose, we here consider the application of the resonant coupling method (RCM) to laser cooling [35,36]. The dissipative force generated by a laser light is known to work only in the longitudinal direction. RCM is employed to extend this powerful 1D cooling force to the transverse degrees of freedom quite easily. All we have to do is simply the excitation of linear coupling resonances. A possible coupling source that induces sufficient correlation between the longitudinal and transverse directions is either a special rf cavity operating in a deflective mode [35] or a regular rf cavity installed at a position with finite dispersion [36]. The two transverse degrees of freedom can be correlated by a solenoid or a skew quadrupole magnet. In order to enhance the efficiency of indirect cooling, the storage ring must operate near difference resonances:

$$
\nu_{x}-\nu_{y} \approx \text { integer, } \quad \nu_{x}-\nu_{z} \approx \text { integer. }
$$

It is obvious that RCM is not limited to laser cooling but can be applied to any general situation where we have one or two dimensional dissipative force that should be extended to the other degree(s) of freedom.

In order to examine how much we can expect from the combination of RCM and the laser-cooling technique, we performed advanced MD simulations in which realistic photon-ion interactions can be incorporated [37]. Table II summarizes main simulation parameters adopted in this subsection. Two cooling lasers, one copropagating and the other counterpropagating with the beam, are introduced in separate straight sections. The three tunes have been chosen so that the resonance conditions above are approximately satisfied. As a source of synchrobetatron coupling, we put a regular rf cavity at a dispersive position. We also turn on a solenoid magnet to couple the horizontal and vertical directions. According to the linear theory in Ref. [36], there is an optimum value for the solenoid coupling. In order to minimize the lattice symmetry breaking, a lower solenoidal field $B_{\text {sol }}$ is preferable. On the basis of the data of test MD simulations, we decided to use $B_{\text {sol }}=40 \mathrm{G}$. Since this field is sufficiently weak, the original sixfold symmetry roughly holds as is clear from Fig. 9.

When the synchrotron tune is much less than the optimum resonant value, no transverse cooling occurs as has been known in past experiments [38]. By moving the operating point near full 3D coupling resonances, we can cool all 3 degrees of freedom simultaneously. Theoretically, it is possible to equalize the damping rates of the three directions by means of RCM [36]. The effectiveness of RCM has been demonstrated in Fig. 10 where

TABLE II. Parameters for laser-cooling simulations.

\begin{tabular}{lc}
\hline \hline Lattice & S-LSR \\
Ion species & $24 \mathrm{Mg}^{+}$ \\
Kinetic beam energy & $35 \mathrm{keV}$ \\
Design superperiodicity $N_{\mathrm{sp}}$ & 6 \\
Bare tunes $\left(\nu_{x}, \nu_{y}, \nu_{z}\right)$ & $(2.067,1.073,0.07)$ \\
Rf harmonic number & 100 \\
Axial length of a solenoid & $0.80 \mathrm{~m}$ \\
Strength of the solenoid field $B_{\mathrm{sol}}$ & $40 \mathrm{G}$ \\
Length of a cooling section & $2.66 \mathrm{~m}$ \\
Transition wavelength $\lambda$ & $280 \mathrm{~nm}$ \\
Saturation intensity & $0.25 \mathrm{~W} / \mathrm{cm}^{2}$ \\
Saturation parameter (on axis) $S_{0}$ & 1.0 \\
Waist radius of lasers $w_{0}$ & $5 \mathrm{~mm}$ \\
\hline \hline
\end{tabular}




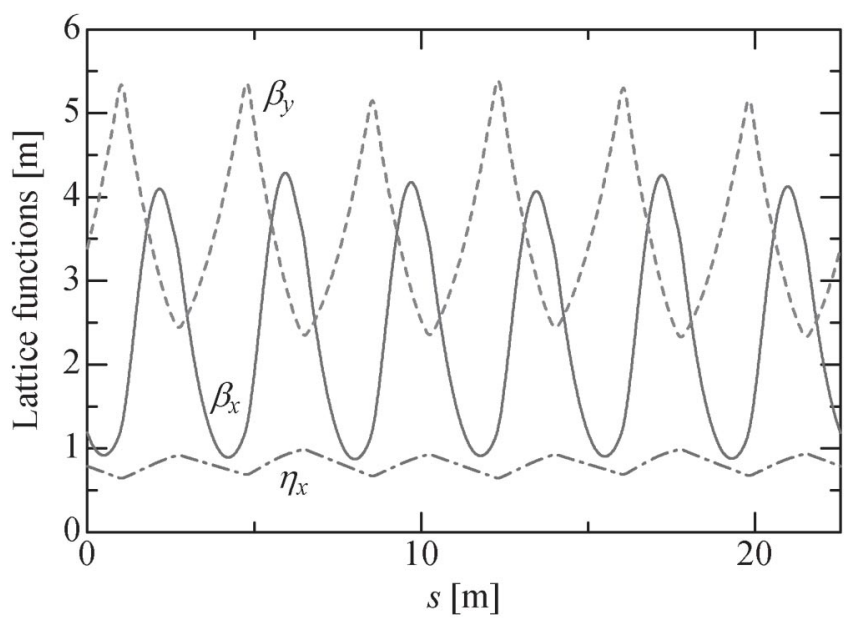

FIG. 9. Lattice functions of S-LSR with a solenoid magnet switched on. $\beta_{x(y)}$ and $\eta_{x}$ are, respectively, the betatron and dispersion functions in the absence of space charge.

we observe dramatic 3D cooling. The normalized rms emittances of the beam have finally reached around $10^{-11} \mathrm{~m}$ rad in all directions. The total number of stored ${ }^{24} \mathrm{Mg}^{+}$ions assumed here is $3 \times 10^{4}$ corresponding to 300 particles in a bunch. The equilibrium distribution of ions after laser cooling has been depicted in Fig. 11. Although the beam is in an ultralow-emittance regime, no clear crystallization can be seen because of the heating mechanisms explained in the last section. The axial distribution of particles in the bunch is not Gaussian but nearly parabolic.

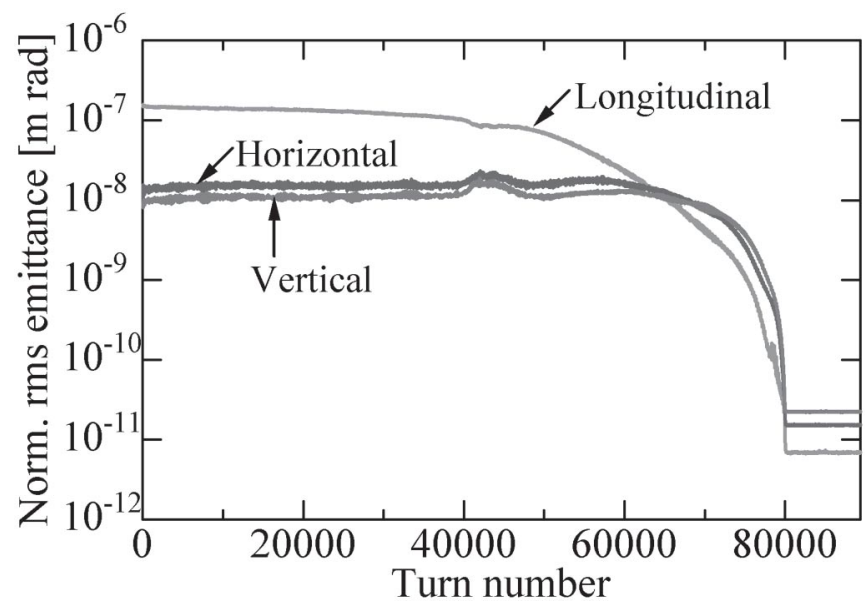

FIG. 10. Time evolution of normalized rms emittances of a ${ }^{24} \mathrm{Mg}^{+}$beam cooled with two counterpropagating lasers in $\mathrm{S}-$ LSR. A regular rf cavity is located in the middle of a straight section where the size of momentum dispersion is $0.8 \mathrm{~m}$. The bare tunes have been adjusted to be $(2.067,1.073,0.07)$ for full $3 \mathrm{D}$ coupling resonances. A scan of the laser frequency is finished at the 80000 th turn. It is possible to make the scan speed faster, but the efficiency of ion capture becomes worsened at high line density due to intrabeam scattering.

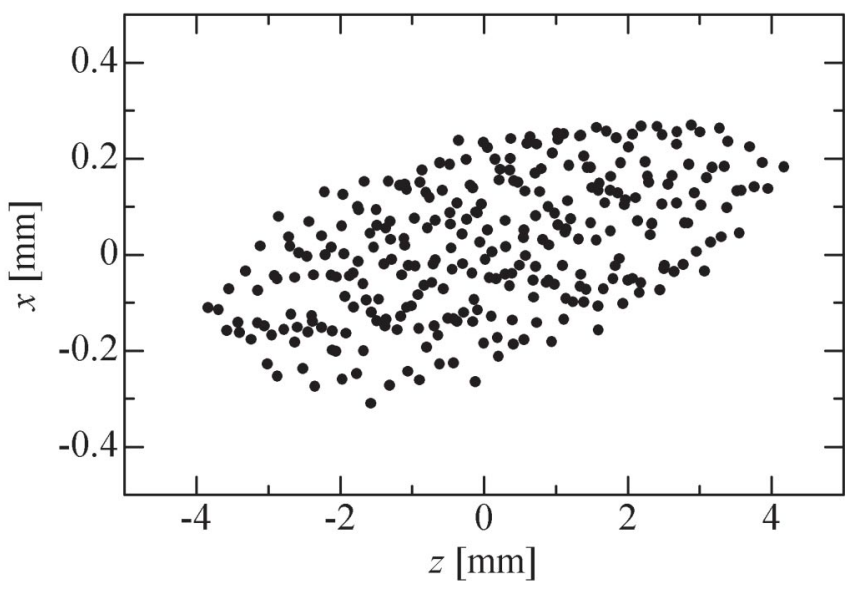

FIG. 11. Real-space configuration (top view) of the lasercooled beam obtained in the MD simulation of Fig. 10.

Since an rf field has been excited, the untracold ellipsoid in Fig. 11 executes the head-tail oscillation exactly periodic in each turn. Figure 12 shows the Fourier spectra calculated from the transverse and longitudinal singleparticle orbits. Before cooling, the dominant Fourier components have the frequencies corresponding to the bare tunes. After cooling, however, the effective betatron tunes are 2.0 in the horizontal direction and 1.0 in the vertical direction [39], while the synchrotron motions of the ions have been completely suppressed. Notice that the tune shifts in the three directions are the same; they are all about 0.07. As the beam is cooled, the effective tunes are gradually depressed by space-charge interactions. Nevertheless, the resonance conditions (6) are approximately retained throughout the cooling process owing to the dispersive oscillation that forces the final incoherent transverse tunes to converge at nonzero round numbers [37]. The normalized rms emittances achievable with RCM are plotted in Fig. 13 as a function of ion number in a bunch. The possible lowest emittance increases due to dispersive heating as we add more particles in a bunch.

With the present simulation setup, a multishell crystalline state cannot be reached not only because the photon pressure is untapered but also because the horizontal phase advance is too large. There is, however, a possibility of attaining a $1 \mathrm{D}$ or $2 \mathrm{D}$ crystalline beam. At $\nu_{z}=0.07$, we expect the formation of a string when the number of ions in a bunch is less than 10. Figure 14(a) shows an MD result where an ion number near this threshold has been taken. We see that, after a laser scanning, ions in each rf bucket are arranged into a linear chain analogous to that in Fig. 6. By adding two more ions, we can convert the string into a vertical zigzag configuration as indicated in Fig. 14(b). A similar structural transition takes place with eight ions when $\nu_{z}$ exceeds 0.09 . These ordered states are fairly stable and can survive for many turns even without the cooling force. 

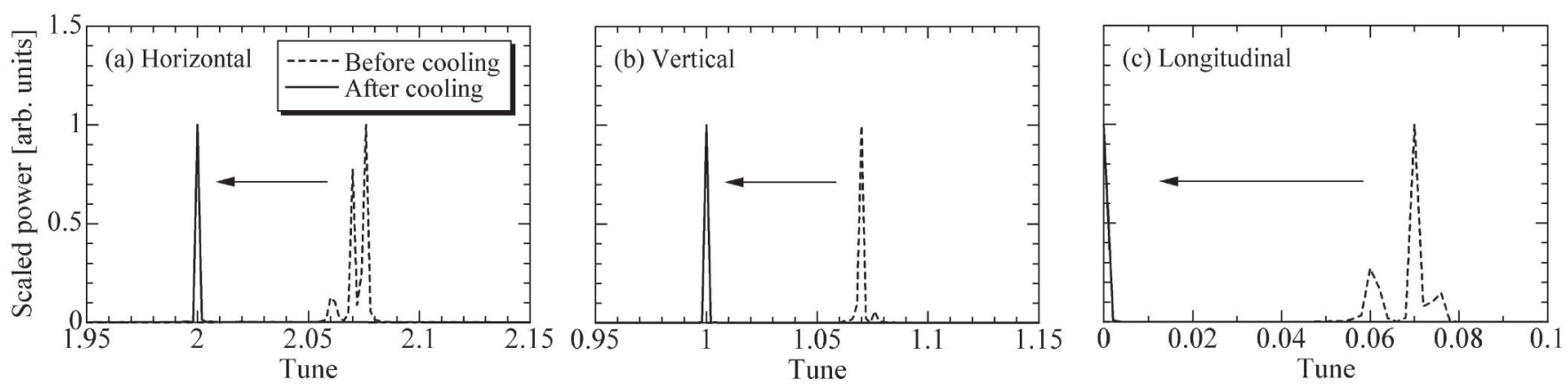

FIG. 12. Fourier spectra of single-particle oscillations before and after laser cooling. The orbit data obtained in the MD simulation of Fig. 10 have been Fourier transformed.

\section{B. Tapered cooling with displaced lasers}

The dissipative force exerted by a single laser on an ion beam operates along the direction of the photon propagation and is expressed as

$$
F=\frac{1}{2} \hbar k_{\mathrm{L}} \Gamma \frac{S}{1+S+(2 \Delta / \Gamma)^{2}}
$$

where $\hbar k_{\mathrm{L}}$ is the photon momentum, $\Gamma$ is the natural line width of the cooling transition, $S$ is the saturation parameter of the laser, and $\Delta$ is the frequency detuning depending on the magnitude of the Doppler shift. Assuming a Gaussian laser for instance, we have an inhomogeneous saturation parameter $S=S_{0} \exp \left[-2\left(x^{2}+y^{2}\right) / w^{2}\right]$ where $S_{0}$ corresponds to the laser intensity on axis, and $w$ is the laser spot size that depends on the longitudinal coordinate. It is evident that the cooling force in Eq. (7) can be tapered by horizontally shifting the laser axis by $\delta x$ [40]. As pointed out in Ref. [9], a tapered force naturally yields horizontal dissipation as well if the cooling section has finite dispersion. We, therefore, anticipate some transverse damping effect free from mutual particle interactions. This idea was experimentally tested at the cooler storage ring in

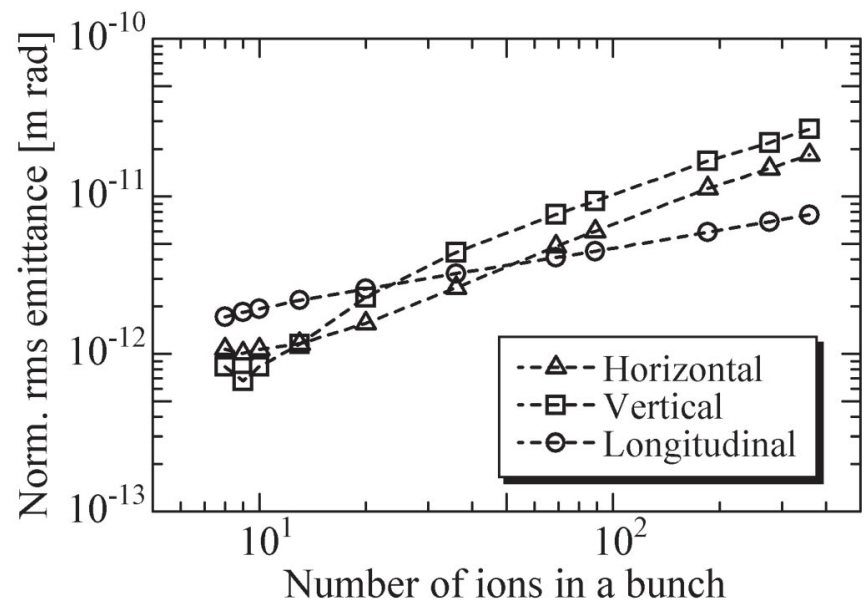

FIG. 13. Final normalized rms emittances of laser-cooled beams vs the number of ions in a bunch.
Heidelberg [40]. The experiment was successful, confirming the indirect transverse cooling by the tapered force [41]. A detailed description of the optimum operating condition and possible cooling rate have been given in Ref. [24].

We can use two [42] or even more displaced lasers [43] to obtain higher flexibility in controlling the tapering co-
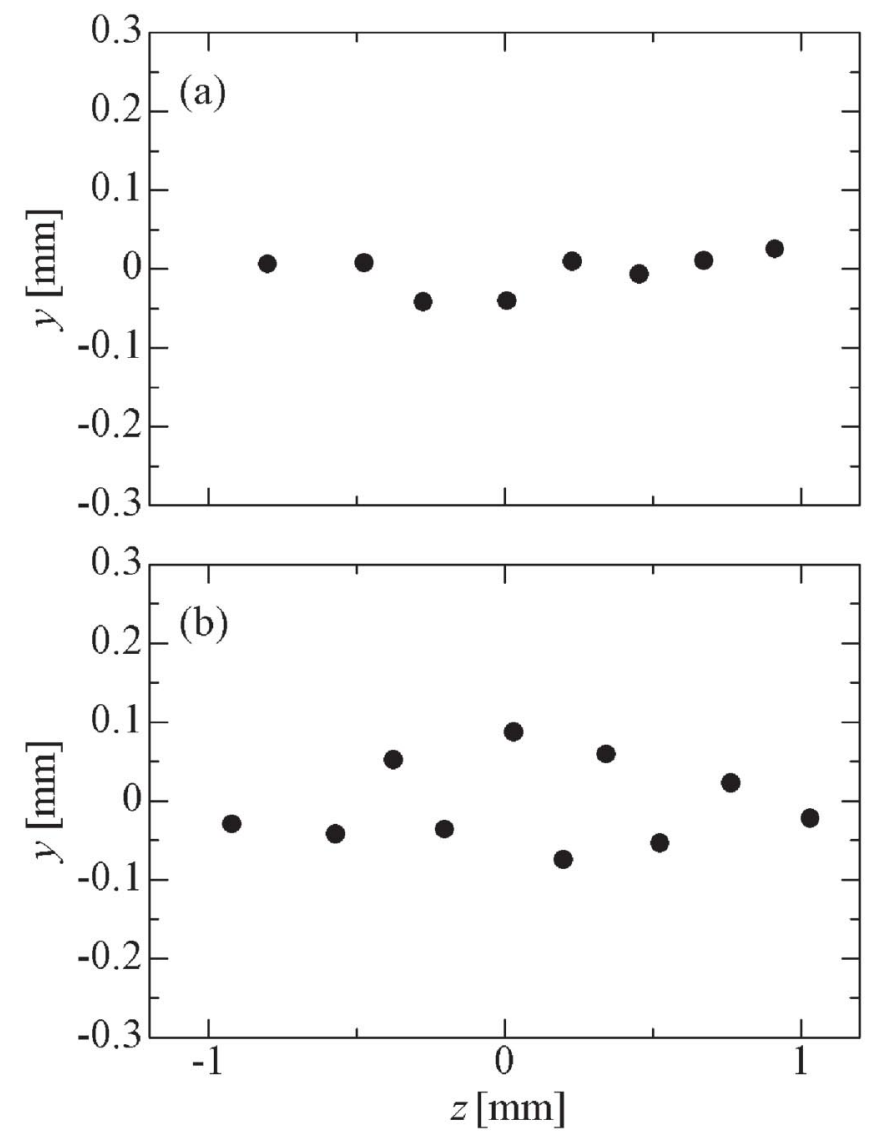

FIG. 14. Real-space configurations (side view) of laser-cooled beams at ultralow intensity. The total number of stored ions is 800 in case (a) and 1000 in case (b). Both beams execute a headtail oscillation about the reference orbit on the horizontal plane. The individual ions do not pass each other longitudinally. 
efficient $C_{x z}$. Expanding the saturation parameter of displaced lasers about the reference orbit and comparing the first-order term in $F$ with the definition (5), we find an approximate tapering factor as

$$
C_{x z} \approx \frac{\Gamma \lambda}{2 \pi} \frac{\rho_{0}}{\beta \gamma^{2} c} \frac{\delta x}{w^{2}} \frac{1+\left(2 \Delta_{0} / \Gamma\right)^{2}}{\left(-2 \Delta_{0} / \Gamma\right)}
$$

where $\lambda$ is the transition wavelength of the ion, $\Delta_{0}$ denotes the detuning for an ion with the design longitudinal velocity, and we have taken into account two counterpropagating lasers symmetrically shifted on the horizontal plane [42]. It turns out that, with a proper detuning and a moderate $\delta x$, the tapering factor realizable by two displaced lasers is on the order of $10^{-2}$ much smaller than the required value of around 0.3 in S-LSR.

Although the use of many displaced lasers with slightly different frequencies enables us to raise $C_{x z}$ up to an ideal level [43], such a complex scheme is not preferred from a practical point of view. In the following MD simulations, therefore, we consider only two cooling lasers for the sake of simplicity and practicability. The lasers are introduced in separate straight sections and horizontally displaced to opposite directions. The average dispersion of the cooling section is about $1.8 \mathrm{~m}$ that should be sufficient to have noticeable transverse cooling effect. A typical time evolution of the beam quality in S-LSR is plotted in Fig. 15 where $\delta x=3 \mathrm{~mm}$ and $\left(\nu_{x}, \nu_{y}\right)=(1.60,1.80)$. We observe a rapid reduction of the longitudinal momentum spread that has reached near $10^{-6}$ at the end of a laser scanning. The horizontal degree of freedom has also been cooled, while nothing occurs in the vertical direction as expected. The best way to make the vertical cooling rate finite is the application of RCM [24]. For this purpose, we first switch on a weak solenoidal field (or a skew quadrupole magnet) and, then, move the operating point onto a transverse coupling resonance [44]. The consequence of this procedure has been shown in Fig. 16. The operating betatron tunes are now $\left(\nu_{x}, \nu_{y}\right)=(2.067,1.073)$ in the absence of space charge. In the case where $\delta x=3 \mathrm{~mm}$, the beam has been cooled three dimensionally and its final configuration is a linear chain as displayed in Fig. 17. Since the tapering factor can never be optimized, it is impossible to form a shell crystalline structure at higher line density. When both lasers are shifted to the other way (i.e., $\delta x=-3 \mathrm{~mm}$ ), the transverse degrees of freedom heats up as predicted by the theory [24]. The experiment in Ref. [40] certainly confirmed this heating mechanism. Note also that the horizontal cooling always takes place after the scan of the laser frequency is almost finished. This is because the transverse cooling rate is too low at a large detuning. We may thus have trouble cooling a beam of high line density with this scheme.

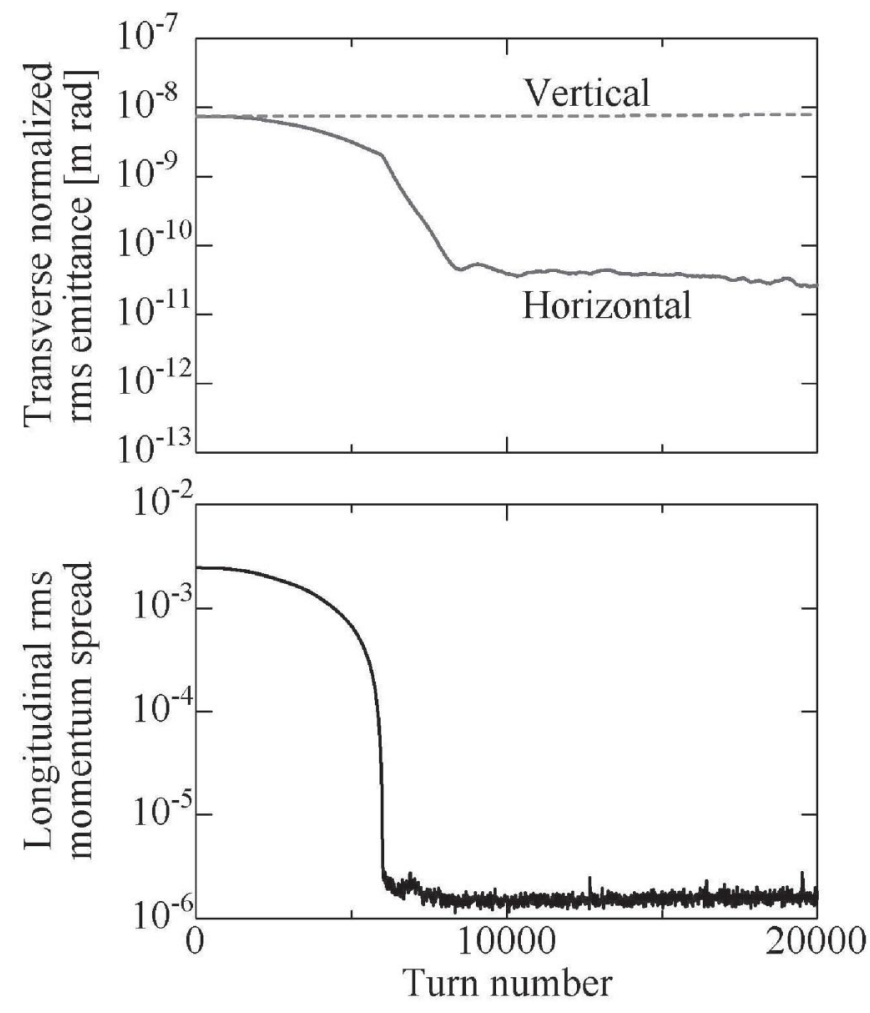

FIG. 15. Time evolutions of the transverse normalized rms emittances and longitudinal rms momentum spread of a $35 \mathrm{keV}^{24} \mathrm{Mg}^{+}$beam cooled with two counterpropagating lasers. Both lasers have been horizontally displaced by $\delta x=3 \mathrm{~mm}$. The line density of the beam is $4.8 \times 10^{3} \mathrm{~m}^{-1}$, and the bare betatron tunes are $\left(\nu_{x}, \nu_{y}\right)=(1.60,1.80)$ in this simulation. The final detuning after a laser-frequency scan completed at 6000th turn is $\Delta_{0}=-75 \mathrm{MHz}$ in the beam rest frame.

\section{SUMMARY}

An ideal crystalline beam circulating in a storage ring possesses the following general characteristics:

(a) The transverse emittances are zero except for quantum noise. The Coulomb coupling parameter is then much higher than the theoretical threshold of 170.

(b) The ordered structure is stable and lasts for many turns without cooling forces.

(c) The orbits of individual particles are periodic and exactly proportional to each other. The periodicity coincides with the lattice periodicity of the ring.

Another important point is, as extensively discussed in the previous sections, whether the beam is exposed to strong momentum dispersion. If dispersion is negligible, the system is physically equivalent to a Paul ion trap in which Coulomb crystallization can quite easily be realized.

In theory, it is possible to crystallize a charged-particle beam even in a dispersive environment if an ideal 3D cooling force is available. The emittance of such an ultimate beam is extremely low in spite of the periodic breathing motion driven by the strong focusing force. In order to establish various crystalline states, the lattice of a storage 


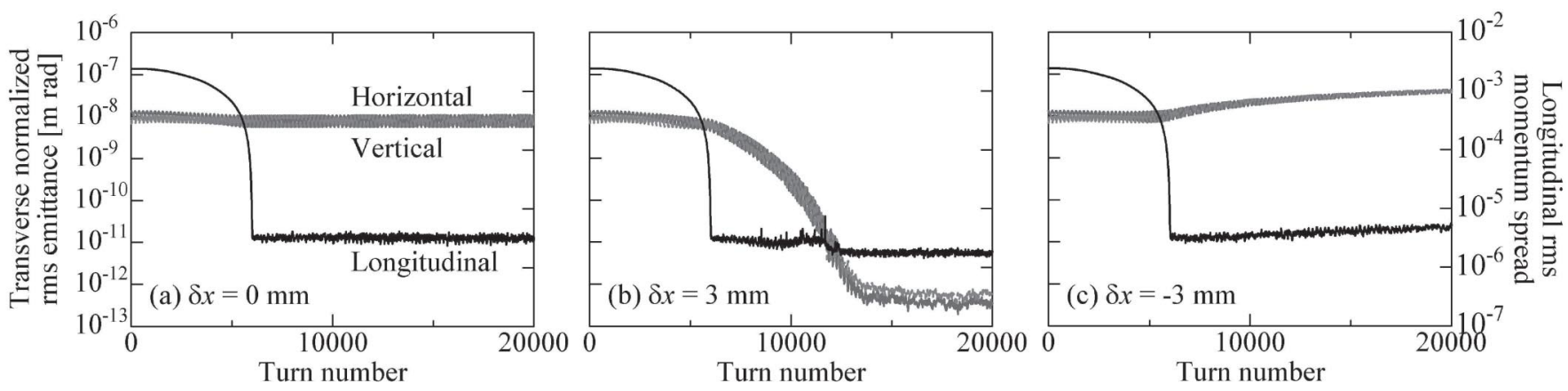

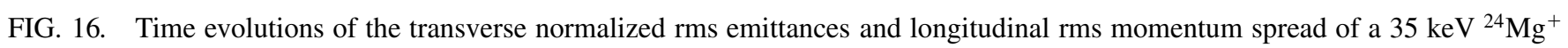
beam cooled with two counterpropagating lasers. The lasers have been horizontally displaced by $\delta x=0 \mathrm{~mm}$ in case (a), $\delta x=3 \mathrm{~mm}$ in case (b), and $\delta x=-3 \mathrm{~mm}$ in case (c). The beam line density and final detuning after a laser scan are the same as assumed in Fig. 15, but the betatron tunes are $\left(\nu_{x}, \nu_{y}\right)=(2.067,1.073)$. The horizontal and vertical cooling (or heating) rates have been equalized by the application of RCM. The transverse coupling source considered here is a solenoid magnet.

ring should satisfy several design criteria; specifically, the ring must operate below the transition energy and have a low betatron phase advance to avoid dangerous resonance crossing. It is also necessary for the cooling efficiency to exceed the rate of heating originating from random Coulomb collisions. The present MD study has demonstrated that the simultaneous use of laser cooling and RCM enables us to attain 1D and 2D crystalline beams. However, the production of a 3D crystalline beam has been found extremely difficult in practice. Most of the difficulties are associated with the existence of momentum dispersion peculiar to a circular system. As clarified by systematic MD simulations, the shear force from bending magnets gives rise to serious complications of the dynamic beam behavior in the ultralow-emittance regime. Since a multishell crystalline beam is very sensitive to external noises, its stability can easily be affected by inevitable sources of perturbation. For instance, even weak lattice asymmetry due to the arrangements of $\mathrm{rf}$ cavities and cooling sections

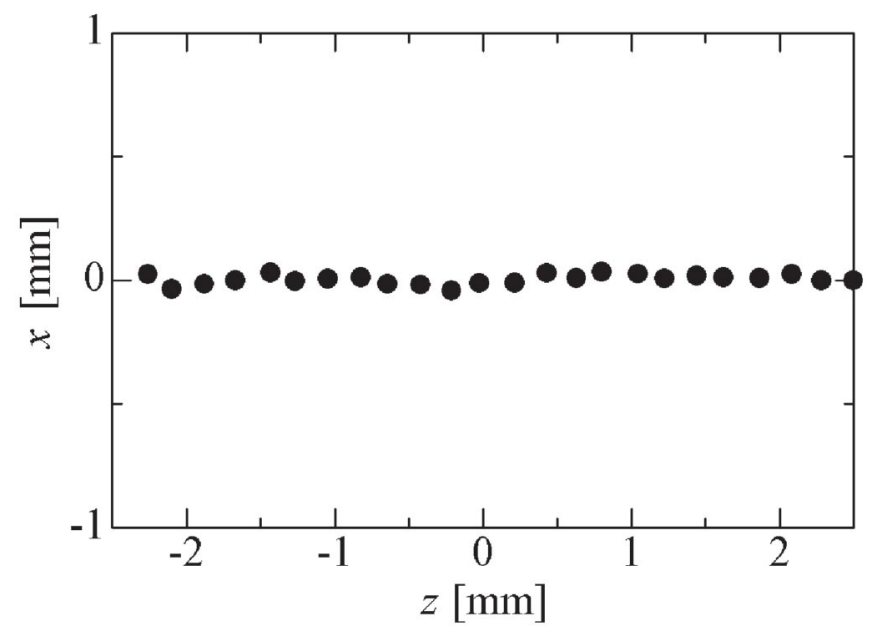

FIG. 17. A coasting string produced by the tapered cooling scheme with displaced counterpropagating lasers. makes it unfeasible to accomplish a 3D crystalline state. Even if the lattice symmetry is strictly maintained, a large crystalline beam can never be stabilized without the tapered force that compensates dispersive heating mechanisms. Considering the sensitivity of a 3D crystalline state as well as the limitation in matching the tapering factor, it seems almost hopeless to generate a stable multishell crystalline beam in reality unless dispersive effects are negligible. The most promising way toward 3D crystallization should probably be the construction of a dispersionless storage ring $[26,27]$ that has high lattice symmetry and low tunes.

\section{ACKNOWLEDGMENTS}

One of the authors (H. O.) would like to thank Andrew Sessler, Jie Wei, and Dieter Möhl for many useful discussions during the course of this work.

[1] Strictly speaking, the emittance of a crystalline beam is finite because of quantum effects. The possible lowest limit of projected emittances should be around $\hbar / m c$ where $\hbar$ is the Planck constant and $c$ is the speed of light.

[2] J. P. Schiffer and P. Kienle, Z. Phys. A 321, 181 (1985).

[3] A. Rahman and J. P. Schiffer, Phys. Rev. Lett. 57, 1133 (1986).

[4] J. Wei, X.-P. Li, and A. M. Sessler, Phys. Rev. Lett. 73, 3089 (1994).

[5] The only exception is a one-dimensionally ordered coasting beam (string crystalline beam) in which all particles are aligned along the design beam orbit.

[6] See, e.g., S. Ichimaru, Rev. Mod. Phys. 54, 1017 (1982).

[7] R. W. Hasse and J. P. Schiffer, Ann. Phys. (N.Y.) 203, 419 (1990).

[8] A. Noda, Nucl. Instrum. Methods Phys. Res., Sect. A 532, 150 (2004).

[9] J. Wei, H. Okamoto, and A. M. Sessler, Phys. Rev. Lett. 80, 2606 (1998). 
[10] An extensive study of phonon modes in various crystalline states has been performed by X.-P. Li et al. See: in Proceedings of the Particle Accelerator Conference, Knoxville, Tennessee, 2005 (to be published).

[11] K. Okabe and H. Okamoto, Jpn. J. Appl. Phys. 42, 4584 (2003).

[12] F. Diedrich et al., Phys. Rev. Lett. 59, 2931 (1987).

[13] D. J. Wineland et al., Phys. Rev. Lett. 59, 2935 (1987).

[14] M. Drewsen et al., Phys. Rev. Lett. 81, 2878 (1998).

[15] H. Okamoto and H. Tanaka, Nucl. Instrum. Methods Phys. Res., Sect. A 437, 178 (1999).

[16] S. Schröder et al., Phys. Rev. Lett. 64, 2901 (1990).

[17] J. S. Hangst et al., Phys. Rev. Lett. 67, 1238 (1991).

[18] H. Okamoto, Phys. Plasmas 9, 322 (2002).

[19] N. Madsen et al., Phys. Rev. Lett. 83, 4301 (1999).

[20] M. Reiser, Theory and Design of Charged Particle Beams (Wiley, New York, 1994).

[21] I. Hofmann et al., Part. Accel. 13, 145 (1983).

[22] H. Okamoto and K. Yokaya, Nucl. Instrum. Methods Phys. Res., Sect. A 482, 51 (2002).

[23] Steck and co-workers reported an anomaly of Schottky signals from electron-cooled heavy-ion beams [M. Steck et al., Phys. Rev. Lett. 77, 3803 (1996)], which was reconfirmed later by Danared et al. [H. Danared et al., Phys. Rev. Lett. 88, 174801 (2002)]. Although this curious event can be interpreted as a longitudinal ordering of stored ions [R.W. Hasse, Phys. Rev. Lett. 83, 3430 (1999)], it is believed to be substantially different from the formation of a string crystalline beam [H. Okamoto et al., Phys. Rev. E 69, 066504 (2004)].

[24] H. Okamoto and J. Wei, Phys. Rev. E 58, 3817 (1998).

[25] As first discussed in Ref. [9] and theoretically formulated in Ref. [24], a longitudinal tapered force simultaneously cools the transverse dimension, provided that the cooling section has finite dispersion. Therefore, this type of frictional force is useful not only for beam crystallization but also for general 3D cooling of a charged-particle beam.

[26] R. E. Pollock, Z. Phys. A 341, 95 (1991).
[27] M. Ikegami et al., Phys. Rev. ST Accel. Beams 7, 120101 (2004).

[28] The spatial configurations of Coulomb crystals in an ordinary ion trap are similar to "bunched" crystalline beams rather than "coasting" crystalline beams.

[29] H. Okamoto, Y. Yuri, and K. Okabe, Phys. Rev. E 67, 046501 (2003).

[30] T. Schätz, U. Schramm, and D. Habs, Nature (London) 412, 717 (2001).

[31] A. M. Sessler (private communication).

[32] U. Schramm, M. Bussmann, and D. Habs, Nucl. Instrum. Methods Phys. Res., Sect. A 532, 348 (2004).

[33] J. Wei and A. M. Sessler, in Proceedings of the 1996 European Particle Accelerator Conference, Sitges, Spain, 1996 (IOP, Bristol, 1996), p. 1179.

[34] Since the tapered force cannot directly cool the vertical betatron motion, we have added weak transverse dissipation in these simulations. Similarly, the crystalline beams in Figs. 1 and 3 have been obtained with a combination of the ideal tapered force and transverse linear friction.

[35] H. Okamoto, A. M. Sessler, and D. Möhl, Phys. Rev. Lett. 72, 3977 (1994).

[36] H. Okamoto, Phys. Rev. E 50, 4982 (1994).

[37] Y. Yuri and H. Okamoto, Phys. Rev. Lett. 93, 204801 (2004).

[38] Only when the beam is initially well conditioned, we observe some "sympathetic" transverse cooling due to Coulomb collisions.

[39] The vertical periodic oscillations are driven by the vertical dispersion created through the solenoid coupling.

[40] I. Lauer et al., Phys. Rev. Lett. 81, 2052 (1998).

[41] The authors of Ref. [40] call this scheme "dispersive cooling" instead of "tapered cooling."

[42] N. Kjærgaard and M. Drewsen, Phys. Lett. A 260, 507 (1999).

[43] T. Kihara, report, 1999 (unpublished).

[44] Essentially the same procedure was taken in the cooling experiment reported in Ref. [40]. 